\title{
A Challenging Case of Non-resolving Pneumonia: Keeping Antisynthetase Syndrome in the Differential Diagnosis
}

Dr. Stephanie Lapinsky MD, Dr. Jessica Leen, MD, Jessica Mak, BSc. Pharm (Hons), RPh, MD Candidate. Dr. Mohammed Shafiee, MD, MSc, FRCPC

\section{About the Author}

Dr. Stephanie Lapinsky, MD, is with the University of Toronto, Department of Obstetrics and Gynecology. Jessica Leen, $M D$ is with the Scarborough and Rouge Hospital, General Internal Medicine. Jessica Mak, BSc. Pharm (Hons), RPh, MD Candidate is with the Faculty of Medicine at the University of Toronto. Dr. Mohammed A. Shafiee MD, MSc, FRCPC, Toronto General Hospital, Division of General Internal Medicine

Correspondence can be directed to: jessica.mak@utoronto.ca

Submitted: March 20, 2018. Accepted: June 10, 2018. Published: February X, 2019. DOI: 10.22374/cjgim.v14i1.278

\begin{abstract}
A 62-year-old Caucasian female ex-smoker presented to the emergency department with progressive shortness of breath with associated pleuritic chest pain, new arthralgias, and muscle weakness for two months. She had already been treated with two courses of antibiotics for suspected community acquired pneumonia with no improvement in her respiratory symptoms. This is a case that illustrates the eventual diagnosis of antisynthetase syndrome, a subtype of the idiopathic inflammatory myopathies. The current standard of diagnostic criteria and treatment for this autoimmune condition will also be discussed.
\end{abstract}

\section{Resume}

Une ex-fumeuse caucasienne âgée de 62 ans s'est présentée à la salle d'urgence avec un essoufflement progressif accompagné de douleurs pleurétiques à la poitrine, de nouvelles arthralgies et une faiblesse musculaire datée deux mois. Elle avait déjà été traitée par deux fois à l'aide d'antibiotiques pour une pneumonie communautaire présumée sans amélioration de ses troubles respiratoires. II s'agit d'un cas qui illustre le diagnostic éventuel du syndrome de l'anti-synthétase, un sous-type des myopathies inflammatoires idiopathiques. La norme actuelle des critères diagnostiques et du traitement pour cette mal adie auto-immune sera également discutée.

A 62-year-old Caucasian female ex-smoker presented to the emergency department with progressive shortness of breath with associated pleuritic chest pain, new arthralgias, and muscle weakness for two months. She had already been treated with two courses of antibiotics for suspected community acquired pneumonia with no improvement in her respiratory symptoms (Figure 1). She denied any recent travel, remarkable environmental or occupational exposures. She had no history of fever, weight loss or other constitutional symptoms. Her past medical history included hypertension, irritable bowel syndrome, and previous diverticulitis. She took no medications. Her brother had severe rheumatoid arthritis.

Upon initial assessment, she was hypoxic (oxygen saturation $85 \%$ on room air and on exertion). Physical examination revealed bibasilar inspiratory crackles, rosacea, tender wrists and metacarpophalangeals (MCPs) bilaterally and symmetrical proximal muscle weakness in both upper and lower extremities.

Her laboratory investigations showed elevated creatinine kinase levels of $3373 \mathrm{U} / \mathrm{L}$ (peak $4795 \mathrm{U} / \mathrm{L}$; Normal range <149U/L), and an erythrocyte sedimentation rate of $40 \mathrm{~mm} / \mathrm{hr}$ (Normal 0-20 $\mathrm{mm} / \mathrm{hr}$ ). She had a mildly elevated troponin level of $0.18 \mathrm{ug} / \mathrm{L}$
$($ Normal $<0.07 \mathrm{ug} / \mathrm{L})$ with an electrocardiogram $(\mathrm{ECG})$ showing evidence of left ventricular hypertrophy but no ischemia and a normal bedside echocardiogram.

Computed tomography (CT) of the chest showed a peripheral and basilar pattern of bilateral pulmonary opacities favouring organizing pneumonia (Figure 2). Subsequent pulmonary

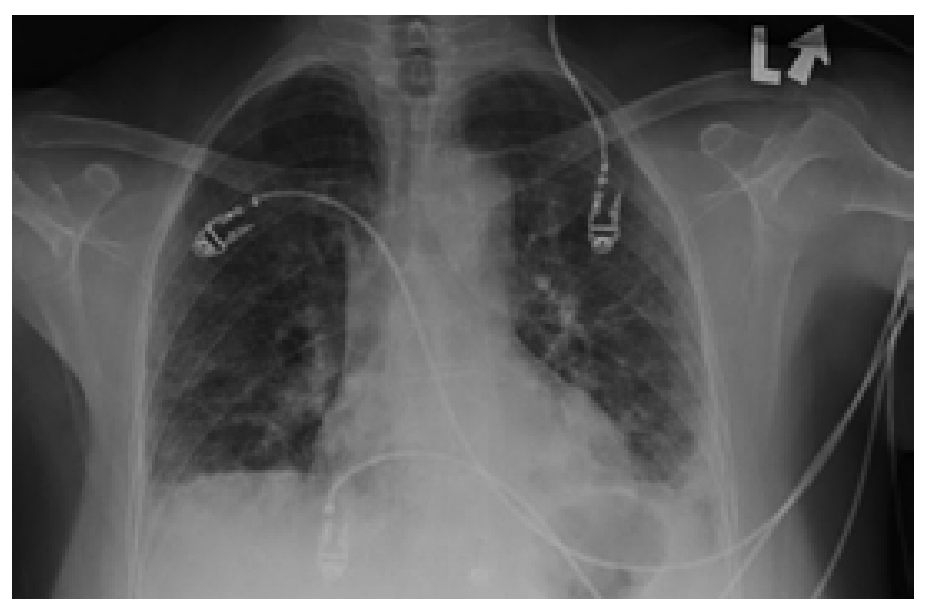

Figure 1. Chest radiograph with opacities and pulmonary edema 


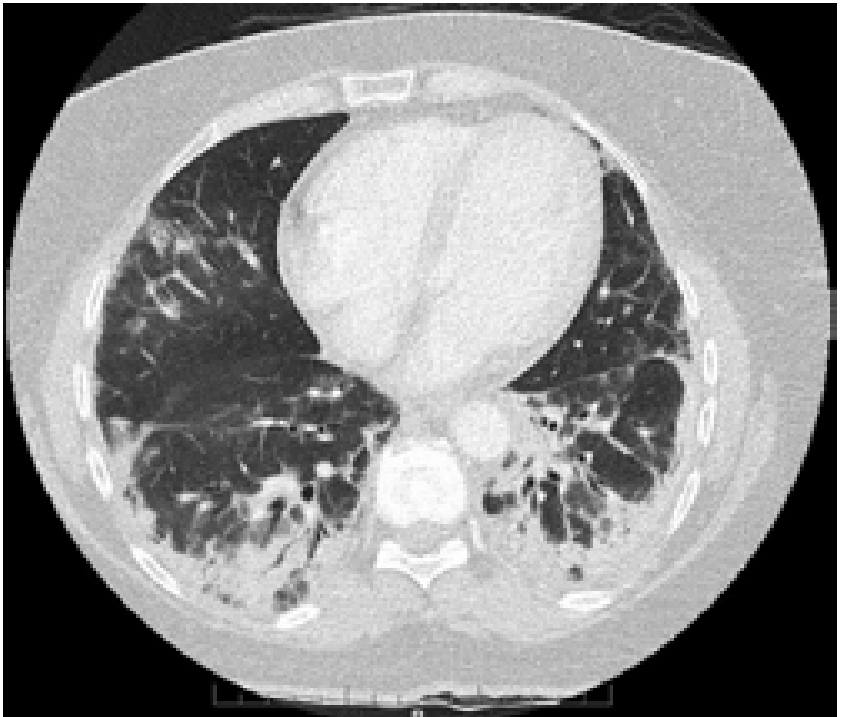

Figure 2. Computed tomography scan of the chest showing bilateral ground glass pulmonary opacities suggesting organizing pneumonia

function testing was symptom-limited but nonetheless, there was a proportional reduction in forced expiratory volume (FEV1) and forced vital capacity (FVC) (51\% and 56\% predicted respectively) and moderate lung restriction with reduced functional residual capacity and residual volume (RV) (66 and 50\% respectively). Malignancy screen (including mammogram and transvaginal ultrasound) was negative.

Possible autoimmune involvement was suspected given her positive family history. She was positive for antinuclear antibodies (ANA). Anti-SSA (Ro) and Anti- Jo-1 titres were both greater than 8.0 AI Units (normal < 0.9 AI Units). Rheumatoid factor, Anti-Cyclic Citrullinated Peptide, C3, C4 and the remainder of the extractable nuclear antibody screen was negative.

Electromyography showed a diffuse myopathic process consistent with an inflammatory myopathy with evidence of muscle fibre inflammation and necrosis. Magnetic resonance imaging of her lower extremities confirmed the presence of diffuse subcutaneous edema consistent with polymyositis.

The positive serology and the electromyography suggested an immune-mediated myopathy at the top of the differential diagnosis. Specifically, the positive Anti-Jo-1, anti-SSA, ANA, respiratory manifestations and myositis, highly favoured the diagnosis of antisynthetase syndrome (AS). Thus, the patient was initiated on high-dose prednisone (60 mg daily) and mycophenolate mofetil $1.5 \mathrm{~g}$ twice a day. She demonstrated symptomatic improvement on the therapy. A left-thigh muscle biopsy was performed one-month post-initiation of treatment and revealed findings consistent with an immune-mediated myopathy and type II fiber atrophy. Finally, a two-month follow-up with a respirologist demonstrated the patient had improved pulmonary function tests (increased vital capacity, improved walk test, and diffusion capacity) from baseline with immunosuppressive therapy.

\section{Pathological Discussion}

The idiopathic inflammatory myopathies (IIMs) are a group of related autoimmune syndromes diagnosed based on a combination of clinical features of skeletal muscle inflammation, characteristic findings on muscle biopsy, and specific autoantibodies. The IIMs include dermatomyositis (DM) and polymyositis (PM), along with other overlapping connective tissue diseases. AS is another systemic autoimmune condition included in this group. AS consists of a constellation of the following symptoms: myositis, arthropathy, fever, Raynaud's phenomenon, mechanic's hands, and interstitial lung disease (ILD), along with the presence of serum autoantibodies against aminoacyl-tRNA synthetases, most commonly the anti-Jo-1 antibody. Other relevant AS antibodies include anti-PL-7 and anti-PL-12. In a patient who presents with both myositis and ILD, an anti-Jo-1 antibody is a very sensitive and specific test for AS.

The clinical presentation of AS is variable, and the precise phenotype is dependent upon the specific autoantibodies present. ${ }^{1}$ In addition, many findings of AS overlap with the findings of other connective tissue disorders and it can take months-to-years for all manifestations of AS to be revealed, leading to delays in diagnosis. ${ }^{2,3}$ Studies have found some correlations between specific autoantibodies and the clinical manifestations presented by AS patients. For instance, Anti Pl-7 is correlated with more severe ILD and myositis is almost always present in those with Anti-Ro/SSA. ${ }^{4}$

Myositis is present in $78-91 \%$ of patients with anti-Jo-1 positive AS, but is usually not the first presenting symptom and may only present after many years of disease. ${ }^{1,5}$ The myositis may present as an isolated elevation of creatine kinase, or can present with clinical symptoms of proximal muscle weakness and pain. The muscles of the esophagus, hypopharynx, and the respiratory muscles can also be involved, leading to a risk of aspiration and shortness of breath. Electromyogram (EMG) studies, MRI of the muscle, and muscle biopsies can all aid in the diagnosis of AS and in monitoring disease progression; however, they are not necessary to make a diagnosis. The findings on muscle biopsy in AS are similar to those seen in other IIMs; however, with some unique features including fragmentation of perimysium connective tissue, perimysium inflammation, and perifascicular atrophy. ${ }^{5,6}$ Arthralgia is present in approximately $75 \%$ of patients with AS, and tends to be asymmetric, non-deforming, non-erosive arthritis, which can present similarly to rheumatoid arthritis. ${ }^{2,3}$ It is the primary presenting symptom in approximately $27 \%$ of patients with AS. ${ }^{2}$

Interstitial lung disease has been shown to be present in $69-90 \%$ of anti-Jo-1 positive patients and is commonly an initial 
presenting symptom, prior to the onset of a myopathy. ${ }^{3,7,8}$ The ILD associated with AS can be severe and is the major cause of morbidity and mortality in patients with AS. It typically presents as exertional dyspnea, often with a non-productive cough. ${ }^{8}$ High-resolution CT scans can be used in the diagnosis of ILD and most commonly show a diffuse, patchy, ground glass opacities and basal consolidations whereas honeycombing and bronchiectasis are seen infrequently., ${ }^{9,10} \mathrm{~A}$ lung biopsy is also useful in the diagnosis and characterization of ILD in AS, with common findings including non-specific interstitial pneumonitis, usual interstitial pneumonitis, cryptogenic organizing pneumonitis or bronchiolitis obliterans organizing pneumonia (COP/BOOP), and diffuse alveolar damage. Spirometry often shows decreased FVC and reduced diffusion capacity, and upright and supine spirometry may reveal evidence of respiratory muscle weakness. When comparing AS to non-AS IIMs, those with AS are more likely to show ILD as the initial presenting symptom, are more likely to be corticosteroid responsive and are more likely to have recurrences of the ILD. ${ }^{8}$ Studies have shown that anti-Jo-1 positive disease tends to have worse pulmonary outcomes, compared to other AS antibodies including anti-PL-12, and the combination of anti-Jo- 1 and anti-Ro (anti-SSA) antibodies tends to be associated with more severe respiratory illness. ${ }^{11}$ Other cardiopulmonary manifestations of AS include pulmonary hypertension, cardiomyopathy, myocarditis, pericardial effusions, and pleural effusions. ${ }^{12}$

Strict diagnostic guidelines for AS is a challenge with such variable clinical presentation for patient cases. Certain features of AS, including Raynaud's, fever, and mechanic's hands, are only seen in a minority of patients and are non-specific findings. The other clinical findings, including myositis, arthralgias, and ILD have been shown more consistently in patients with AS. Soloman et al provided stricter diagnostic criteria in 2011 with a diagnosis of AS being the presence of tRNA synthetase auto-antibodies and at least one major manifestations (myositis, dermatomyositis, ILD) and at least two minor manifestations (mechanic's hands, Raynaud's, arthritis, unexplained fever). ${ }^{13}$

Because AS is associated with high morbidity, prompt diagnosis and treatment is important, especially as it is generally responsive to immunomodulatory therapy. There are no controlled trials of the treatment of AS, however, the recommended first-line treatment of AS involves corticosteroids at a dose of $1 \mathrm{mg} / \mathrm{kg} /$ day, tapered after 6-8 weeks. Treatment can also involve steroidsparing immunomodulatory agents, such as cyclophosphamide, azathioprine, mycophenolate mofetil, cyclosporine, tacrolimus, rituximab, and IVIG. One case report of refractory AS after a trial with a number of immunosuppressive treatments showed remission with 4 months of treatment with mycophenolate mofetil (MMF) at a dose of $2 \mathrm{~g} /$ day, which was well-tolerated. ${ }^{14}$
Although case reports and case series have shown success in treating AS with these agents, it has also been shown that AS has a tendency to relapse and can be severe and unresponsive to treatment. ${ }^{9}$ Thus relapse is another important factor to monitor for in AS patients who are tapered or discontinued off immunotherapy. Predictors of poor prognosis include older age of onset, malignancy, and negative immunologic tests. ${ }^{15}$ Management of AS should also include a malignancy screen as there exists an association between various malignancies and AS. It is unclear whether these malignancies may play a causal role in the development of AS through a paraneoplastic process. Appropriate clinical precautions applicable to patients on longterm immunosuppressants such as vaccination, Pneumocystis Jiroveci Carinii pneumonia prophylaxis and management of adverse effects (e.g., osteoporosis prevention with glucocorticoids) also applies for AS patients. ${ }^{16}$

\section{Conclusions}

\section{Antisynthetase Syndrome}

AS is a clinically heterogenous small subset of DM-PM which can present with isolated respiratory symptoms such as cough and shortness of breath with associated, delayed or absent muscle symptoms. It should be considered in the differential of nonresolving pneumonia.

\section{Initial Symptoms and Clinical Presentation}

- Initial symptoms: dyspnea, cough, fever, muscle weakness

- DM-PM-like muscle weakness, myalgias, arthralgias or skin changes can follow months later

- Major manifestations: myositis, dermatomyositis, ILD

- Minor manifestations: mechanic's hands, Raynaud's, arthritis, unexplained fever

\section{Important Diagnostic Tests with Reported Prognostic Value}

- Diagnostic tests can aid in early diagnosis and therefore early treatment

- Markers of muscle inflammation (CK) and autoantibodies against aminoacyl-tRNA synthetases (especially anti-Jo-1, Anti-PL12/7)

- Presence of Anti-SSA/SSB antibodies

- Respiratory investigations including high-resolution CT scan (pattern and extent of disease); bronchoscopy with bronchoalveolar lavage and lung biopsy are often not required for diagnosis but can be considered in unclear cases to confirm the diagnosis.

- Pulmonary function testing for diffusion capacity, and maximum inspiratory/expiratory pressure (MIPS/MEPS) to assess respiratory muscle involvement 


\section{Also, Consider Within Diagnostic Workup}

- Echocardiogram to screen for pulmonary hypertension

- Rule out malignancy: ultrasound, imaging

\section{Treatment}

- No formal guidelines currently for a standard of treatment, patients often initiated on high-dose glucocorticoids and immunosuppressants

- Monitor for relapse in patients particularly after tapering glucocorticoids

- Even with appropriate treatment, including high-dose glucocorticoids and immunosuppressants (e.g. MMF), respiratory symptoms may persist and are the major cause of morbidity and mortality (up to $40 \%$ increase in mortality). This may require long-term supportive care, including home oxygen and referral to an outpatient respirologist.

\section{Funding Statement}

None.

\section{Competing Interests}

None to declare.

\section{References}

1. Katzap E, Barilla-LaBarca M, Marder G. Antisynthetase Syndrome. Curr Rheumatol Rep 2011;13:175-181.

2. Lefevre G, Meyer A, Launay D, et al. Seronegative polyarthritis revealing antisynthetase syndrome: a multicentre study of 40 patients. Rheumatology (Oxford). 2014; Epub.

3. Lega JC, Fabien N, Reynaud Q, et al. The clinical phenotype associated with myositis-specific and associated auto-antibodies: a meta-analysis revisiting the so-called antisynthetase syndrome. Autoimmun Rev 2014;13(9):883-91.
4. Dugar M, Cox S, Limaye V, Blumbergs P, Roberts-Thomson PJ. Clinical heterogeneity and prognostic features of South Australian patients with antisynthetase autoantibodies. Int Med J 2011:674-9.

5. Schmidt WA, Wetzel W, Friedländer R, et al. Clinical and serological aspects of patients with anti-Jo-1 antibodies - an evolving spectrum of disease manifestations. Clin Rheumatol 2000;19(5):371-7.

6. Mozaffar T, Pestronk A. Myopathy with anti-Jo-1 antibodies: pathology in perimysium and neighbouring muscle fibres. J Neurol Neurosurg Psychiatr 2000;68(4):472-8.

7. Targoff IN. Update on myositis-specific and myositis-associated autoantibodies. Curr Opin Rheumatol 2000;12(6):475-81.

8. Richards TJ, Eggebeen A, Gibson K, et al. Characterization and peripheral blood biomarker assessment of anti-Jo-1 antibodypositive interstitial lung disease. Arthritis Rheum 2009;60(7):2183-92.

9. Yoshifuji H, Fujii T, Kobayashi S, Imura Y, Fujita Y, Kawabata D, Usui T, Tanaka M, Nagai S, Umehara H, Mimori T. Anti-aminoacyl-tRNA synthetase antibodies in clinical course prediction of interstitial lung disease complicated with idiopathic inflammatory myopathies. Autoimmunity 2006;39(3):233-41.

10. Ingegnoli F, Lubatti C, Ingegnoli A, Boracchi P, Zeni S, Meroni PL. Interstitial lung disease outcomes by high-resolution computed tomography (HRCT) in Anti-Jol antibody-positive polymyositis patients: A single centre study and review of the literature. Autoimmun Rev 2012;11:335-40.

11. Schnabel A, Reuter M, Biederer J, et al. Interstitial lung disease in polymyositis and dermatomyositis: clinical course and response to treatment. Semin Arthritis Rheum 2003;32(5):273-84.

12. Kalluri M, Oddis CV. Pulmonary manifestations of the Idiopathic Inflammatory Myopathies. Clin Chest Med 2010;31:501-12.

13. Witt LJ, Curran JJ, Strek ME. The Diagnosis and Treatment of Antisynthetase Syndrome. Clin Pulm Med 2016 Sep;23(5):218-26.

14. Sharma K, Orbai AM, Desai D, et al. Brief report: Antisynthetase SyndromeAssociated Myocarditis. J Card Fail 2014;Epub.

15. Hervier B, Masseau A, Mussini JM, Audrain M, Hamidou MA. Long-term efficacy of mycophenolate mofetil in a case of refractory antisynthetase syndrome. Joint Bone Spine 2009;76(5):575-6.

16. Public Health Agency of Canada. PHAC: Canadian Immunization Guide section 3 - Vaccination of specific populations (acquired/secondary immunodeficiency). Available at: http://www.phac-aspc.gc.ca/publicat/ciggci/p03-07-eng.php\#a4 